\title{
ÍNDICE DE VALOR ECOLÓGICO (IES) COMO FERRAMENTA PARA ESTUDOS FITOSSOCIOLÓGICOS E CONSERVAÇ̃̃O DAS ESPÉCIES DE MUSGOS NA BAIA DO ALMIRANTADO, ILHA REI GEORGE, ANTÁRTICA MARÍTIMA
}

\author{
Filipe de Carvalho Victoria ${ }^{1, *} \&$ Antônio Batista Pereira ${ }^{2}$ \\ ${ }^{1}$ Núcleo de Estudos da Vegetação Antártica, NEVA, Rua Gonçalves Chaves 412/503, CEP: 96015-560, Pelotas, RS, Brasil. \\ ${ }^{2}$ Universidade Estadual do Rio Grande do Sul, Rua Dr. José Bisognin, 250, CEP: 99700-000, Erechim, Rio Grande do Sul, Brasil. \\ *E-mail: filipevictoria@gmail.com
}

\section{RESUMO}

A distribuição das comunidades vegetais antárticas é dependente das condições ambientais e também das condições pedogeomorfológicas, como estabilidade da superfície, tipo de rocha e erosão eólica. São estabelecidos nítidos limites de sobrevivência, fazendo com que certas espécies de musgos sejam altamente especializadas à geomorfologia local. Durante os verões austrais 2003/2004 e 2004/2005 foram realizados estudos fitossociológicos, nas áreas de degelo adjacentes a Estação Polonesa Henri Arctowski e em Ponta Hennequim, ambas na Baía do Almirantado, Ilha Rei George, objetivando estudar a estrutura das comunidades de musgos nas áreas de degelo. Neste trabalho analisamos as ameaças potenciais às espécies de musgos mais freqüentes nestas comunidades utilizando o índice de valor ecológico (IES) como ferramenta de apoio aos estudos fitossociológicos e de conservação das espécies. Das 58 espécies de musgos conhecidas para a Baía do Almirantado, 19 ocorrem com maior freqüência. Sanionia uncinata (Hedw.) Loeske e Polytrichastrum alpinum (Hedw.) G. L. Smith aparecem como as espécies menos ameaças e Bryum amblyodon Müll. Hal., Ditrichum hyalinum Mitt. Kuntze, Pohlia drumondii (Müll. Hal.) A.L. Andrews e Schistidium falcatum (Hook. f. et Wils.) B. Bremer as mais ameaçadas pela presença humana na região estudada

Palavras-chaves: Fitossociologia, espécies ameaçadas, ação antrópica.

\begin{abstract}
INDEX OF ECOLOGICAL SIGNIFICANCE (IES) AS A TOOL TO PHYTOSSOCIOLOGICAL AND CONSERVATION STUDIES FOR MOSS SPECIES IN ADMIRALTY BAY AREA, KING GEORGE ISLAND, ANTARCTICA. The Antarctic plant distribution depends on the environmental factors, like geomorphological conditions, as surface's stability, rock type and aeolian erosion. It establishes clear home ranges, leading some mosses species to be highly correlated to local geomorphology. During the austral summer 2003/2004 and 2004/2005 we carried out ecological studies at the ice-free areas adjoining the Polish Station Henri Arctowski and the Point Hennequim localities, both in the Admiralty Bay, inside of the King George Island, to study community structure of mosses in these areas. This work presented potentials threats for the more representative moss species in these region, using the ecological value index as a tool for phytosociological and conservation studies. Ninenth, for fifty-eigth species of mosses know for Admiralty Bay area occur more frequently. Sanionia uncinata (Hedw.) Loeske and Polytrichastrum alpinum (Hedw.) G. L. Smith show as low threats and Bryum amblyodon Müll. Hal., Ditrichum hyalinum Mitt. Kuntze, Pohlia drumondii (Müll. Hal.) A.L. Andrews and Schistidium falcatum (Hook. f. et Wils.) B. Bremer appear as the most threatened species, especialy by the human presence in the region.
\end{abstract}

Key-words: Phytosociology; threatened species, human impacts.

\section{INTRODUÇÃO}

A Ilha Rei George $\left(61^{\circ} 50^{\prime} \mathrm{S}, 62^{\circ} 15^{\prime} \mathrm{W}\right)$ está localizada no Arquipélago Shetlands do Sul (Antártica Marítima). No interior da ilha localiza-se a Baía do Almirantado, uma região que por ser abrigada, apre- senta um microclima bastante distinto ao encontrado em outras partes da ilha, especialmente em relação aos ventos (Lewis-Smith 1972, Putzke \& Pereira 2001). Em consideração a intensa atividade humana, foi criada nesta região a Área Antártica Especialmente Gerenciada (Antarctic Special Managed Area-AS- 
MA) da Baía do Almirantado, objetivando minimizar o impacto sobre a biota (Simões et al. 2001).

Segundo Lewis-Smith (2001), desde 1940 são observadas evidências do aquecimento global nas plantas da Antártica marítima, principalmente em relação às oscilações na percentagem da cobertura vegetal. Uma ferramenta importante para estudar as flutuações nas populações de briófitas foi idealizada por Lara \& Mazimpaka (1998). Estes autores desenvolveram o Índice de valor ecológico (Index of Ecological Significance-IES), que compara os dados de freqüência e a abundância, determinando a importância das espécies na área estudada. Este índice pode ser aplicado em estudos fitossociológicos e de conservação de espécies de musgos, tendo sido utilizado por Marques et al. (2005) em estudos comparativos de importância de briófitas em duas formações vegetais distintas na Europa. Estes autores evidenciam que análises fitossociológicas, utilizando como ferramenta o índice de valor ecológico, podem ser aplicados na classificação de espécies ameaçadas (Hallingbäck et al. 1996, Hallingbäck \& Hodgetts 2000), proposta pela União Internacional para a Conservação da Natureza (International Union for Conservation of Nature-IUCN).

Além de refletir a importância da espécie em determinada formação vegetal, este índice também demonstra o grau de associação desta com outras espécies na amostragem, bem como o grau de importância desta para a manutenção da formação. Muitas espécies estão associadas com as espécies dominantes da formação e só ocorrem desta maneira, isto invariavelmente acaba refletindo a dependência das espécies de musgos associadas e, se as espécies dominantes estiverem ameaçadas de alguma forma, suas dependentes também estarão (Lewis-Smith 2001).

Este índice também indica o grau de colonização de determinada espécie na amostra (Lara \& Mazimpaka 1998), refletindo as variações de ocorrência das espécies nos substratos disponíveis. Assim, se uma espécie depende de substratos ricos em matéria orgânica e estes estiverem em menor quantidade na região, as espécies colonizadoras estarão decaindo na medida em que este substrato desaparece (LewisSmith 1990, Victoria et al. 2006).

O objetivo deste trabalho foi demonstrar como o IES pode ser útil nas análises de impacto das alterações ambientais e das atividades humanas sobre as comunidades vegetais da Antártica Marítima, a partir de um ensaio com algumas espécies de musgos que ocorrem nas áreas de degelo da Baía do Almirantado.

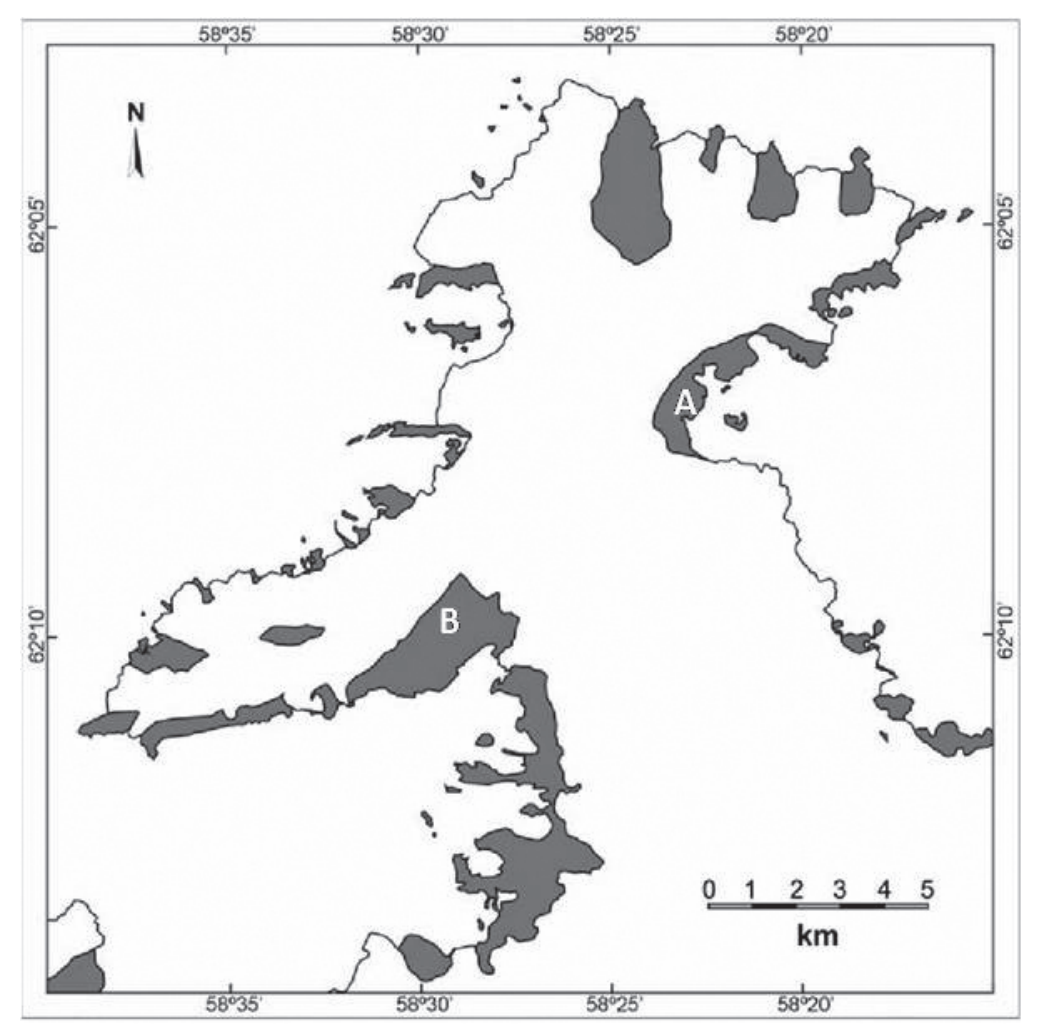

Figura 1. Baía do almirantado, Ilha Rei George, Arquipélago Shetlands do Sul, Antártica. Áreas estudadas. A) Ponta Hennequin. B) Região de Arctowski (modificado de Simões et al. 2004). 


\section{MATERIAL E MÉTODOS}

No presente trabalho foram utilizados os dados obtidos em estudos fitossociológicos realizados na Baía do Almirantado durante o verão austral 2003/2004 e 2004/2005, nas adjacências do Refúgio Equatoriano na Ponta Hennequin $\left(62^{\circ} 08 \mathrm{~S}, 58^{\circ} 24 \mathrm{~W}\right)$ e na região da estação polonesa Henryk Arctowski $\left(62^{\circ} 09 \mathrm{~S}, 58^{\circ} 27 \mathrm{O}\right)$ distantes cerca de $7 \mathrm{Km}$ uma da outra, separadas pelas águas da baía (Figura 1). Estas áreas foram escolhidas para este estudo por apresentarem as maiores manchas de vegetação da Baía do Almirantado (Ochyra 1998), possibilitando amostrar o maior número de espécies e conseqüentemente o quanto estas são freqüentes na ASMA da Baía do Almirantado.

Foram dispostos 526 quadrados nas regiões estudadas, sendo 240 na região de Arctowski e 286 em Ponta Hennequin. Nos quadrados foram avaliados o grau de cobertura e a freqüência contando a porcentagem de área que as espécies ocupavam no quadrado, utilizando-se o método de quadrados de Braun-Blanquet (1932). O grau de cobertura foi medido em cada quadrado individualmente, segundo Kanda (1986), com adequações ao método de BraunBlanquet (1932).

As espécies de musgos que apresentaram maior percentual de cobertura na abordagem fitossociológica foram selecionadas para verificação do status de conservação destas nas áreas estudadas, com base na importância ecológica de cada uma.

Para ilustrar a importância ecológica das espécies na amostragem total, foi utilizado o índice de valor ecológico (Lara \& Mazimpaka 1998), que combina os parâmetros de abundância (cobertura e freqüência) sendo o índice assim discriminado (Marques et al. 2005):

$$
\mathrm{IES}=\mathbf{F}(\mathbf{1}+\mathbf{C})
$$

Onde:

F é a freqüência relativa da espécie na área ou habitat, é dado pelo número de ocorrências (x) dividido pelo número total das amostras consideradas (n): $\mathbf{F}=\mathbf{1 0 0 x} / \mathbf{n}$

C é a cobertura média da espécie nas amostras: $\mathbf{C}=\Sigma\left(\mathbf{c}_{\mathrm{i}}\right) / \mathbf{x}$; onde $\mathrm{c}_{\mathrm{i}}$ é a classe de cobertura e x o número de pontos amostrais que a espécie ocorre, são similares aos usados por Braun-Blanquet (1932).

A escala de valores de índice varia de 0 a 600 , mas na prática, e de acordo com seus autores, os valores acima de 400 são muito raros, desde que representam um domínio consistente e quase absoluto de um táxon. No geral, os valores acima de 50 revelam uma importância ecológica significativa. As vantagens de usar este índice são a sua simplicidade, porque usa valores da freqüência relativa, e o fato de que uma sobre-avaliação da freqüência relativa evita a distorção causada

Tabela I. Espécies de musgos mais freqüentes nas comunidades vegetais em áreas de degelo da Baía do Almirantado e sua importância ecológica indicada pelo Índice de Valor Ecológico.

\begin{tabular}{|c|c|c|c|}
\hline EsPÉCIES & $\mathrm{F}(\%)$ & $\mathrm{C}$ & IES \\
\hline Sanionia uncinata (Hedw.) Loeske & 57,87 & 2,7 & 215,20 \\
\hline Polytrichastrum alpinum (Hedw.) G. L. Smith & 31,86 & 3,8 & 153,54 \\
\hline Andreaea gainii Card. & 14,96 & 0,6 & 24,54 \\
\hline Polytrichum juniperinum Hedw. & 11,36 & 2,02 & 34,3 \\
\hline Bartramia patens Brid. & 1,71 & 0,6 & 2,03 \\
\hline Bryum palliescens Schelich. ex Schwägr. & 1,47 & 0,38 & 2,03 \\
\hline Schistidium antarctici (Card.) Savicz. et Smirn & 1,33 & 0,36 & 1,87 \\
\hline Bryum orbiculatifolium Card \& Broth. & 0,95 & 0,74 & 1,66 \\
\hline Andreaea depressinervis Card. & 0,19 & 5,58 & 1,25 \\
\hline Bryum amblyodon Müll. Hal. & 0,19 & 3,31 & 0,83 \\
\hline Ditrichum hyalinum Mitt. Kuntze & 0,19 & 3,31 & 0,83 \\
\hline Pohlia drumondii (Müll. Hal.) A.L. Andrews & 0,19 & 3,31 & 0,83 \\
\hline Schistidium falcatum (Hook. f. et Wils.) B. Bremer & 0,19 & 3,31 & 0,83 \\
\hline
\end{tabular}

( $\mathrm{F}=$ freqüência das espécies em 526 quadrados amostrados; $\mathrm{C}=$ cobertura média da espécie na amostragem; IES=Índice de valor ecológico) 
pela presença de táxons ocasionais, mas com maior cobertura na amostragem, que podem apresentar valores mais elevados do que aqueles mais freqüentes, mas com menor cobertura (Lara \& Mazimpaka 1998).

Para a identificação das briófitas utilizou-se Putzke \& Pereira (1990), Ochyra (1998), Bednarek-Ochyra et al. (2000), Putzke \& Pereira (2001).

O mapa e os nomes das localidades apresentadas neste trabalho seguem a nomenclatura oficial de acordo com Simões et al. (2004).

\section{RESULTADOS E DISCUSSÃO}

A partir dos dados obtidos na amostragem fitossociológica foi possível evidenciar 19 espécies mais freqüentes de 58 espécies de musgos que ocorrem nas comunidades vegetais das áreas de degelo da Baía do Almirantado (Tabela I).

De acordo com o IES de cada espécie, foi possível verificar que a maioria das espécies é facilmente encontrada nas regiões, porém grande parte com baixa cobertura (IES $>50$ ). Isto pode significar que estas espécies são mais sensíveis às alterações no ambiente, uma vez que, possuem populações pequenas com menor resistência e resiliência, pois as inter-relações entre os organismos são reduzidas (Schaefer et al. 2004), sendo que os impactos causados nestas pode não ser reversível.

Como exemplo, podemos citar as espécies do gênero Bryum e Andreaea. Espécies como Bryum pseudotrichetum e Bryum pallescens são altamente dependentes das linhas d'agua formada pelo degelo sazonal no verão austral (Allison \& Lewis-Smith 1973, Kanda 1986, Kanda \& Ohtani 1991). Bryum pseudotrichetrum possui maior abundância nas amostras estudadas no presente trabalho e por isso pode ser considerada como uma espécie em menor grau de ameaça se comparado a $B$. pallescens devido ao maior tamanho na população (Lewis-Smith 1990), que possibilita maiores chances de sucesso adaptativo em caso de mudanças bruscas no ambiente onde ocorrem (Lewis-Smith 2001).

As espécies do gênero Andreaea ocorrem principalmente em afloramentos rochosos e dependem da rocha nua para se fixarem (Ochyra 1998, Hu 1998, Putzke \& Pereira 2001). Geralmente a atividade de pesquisadores e militares nas formações vegetais da Baía do Almirantado causa danos aos afloramentos de rocha durante o deslocamento nas áreas de degelo. Fragmentos da rocha se desprendem facilmente dos afloramentos, limitando área de colonização para algumas espécies de musgos como o exemplo acima.

Estas são comumente encontradas nas praias e terraços marítimos da Baía do Almirantado. Os substratos nestas áreas são ricos em matéria orgânica, devido principalmente à atividade ornitogênica (Michell et al. 2006). Assim sendo, estas plantas possuem dependência das colônias de aves e as oscilações na população de aves podem afetar as comunidades vegetais na região.

Segundo Ochyra (1998) Sanionia uncinata e Polytrichastrum alpinum são as espécies de maior abundâncianaAntártica Marítima, estandoassimmenos ameaçadas em relação às demais espécies selecionadas neste estudo. Isto também foi observado no presente estudo, uma vez que ambas as espécies apresentaram os maiores índices de valor ecológico (IES $=215,20$ e IES=153,54 sucessivamente). Estas duas espécies ocorrem em substratos rico em matéria orgânica, nas praias e terraços marítimos, preferencialmente próximos às colônias de aves (Kanda 1986, Putzke \& Pereira 2001). Portanto a permanência destas espécies esta diretamente relacionada com o grau de ameaça que as aves migratórias estejam sujeitas.

Outras espécies como A. depressinervis, B. patens e $S$. antarctici ocorrem preferencialmente em áreas de predomínio de afloramentos rochosos, onde o substrato é facilmente deslocado, seja pela ação da água de degelo, seja pela ação dos ventos (Longton 1988). Olech (1996), relata que esta circunstância da dinâmica do substrato em ecossistemas polares pode ser agravada pela intervenção humana no meio terrestre da Antártica Marítima. Este fato evidencia o nível de ameaça os quais estão sujeitas as espécies de musgos que colonizam este tipo de substrato, refletida na baixa freqüência e menores valores de IES para estas espécies (IES $>2$ ).

Algumas espécies como S. falcatum ocorrem sobre seixo e rocha solta pela ação dos liquens, dependendo de associações com estes para se manterem nas comunidades (Victoria et al. 2006). O maior impacto neste tipo de substrato é causado pela atividade humana (Olech 1996, Lewis-Smith 2001). A presença de militares e pesquisadores nas adjacências das estações de pesquisa na Baía do Almirantado modifica o substrato e pode dificultar 
a colonização dos liquens e conseqüentemente das espécies de musgos associadas a estes. No caso da espécie de musgo acima, este fato é ainda mais perturbador, uma vez que esta espécie encontra-se ameaçada por estar perdendo o substrato preferencial de colonização e ocorrer em baixa freqüência na região (IES $=0,83$ ).

Todas estas espécies de musgos, bem como a biota terrestre da Baía do Almirantado, são diretamente e indiretamente afetadas pela presença do homem. A manutenção da vida de pesquisadores e militares dentro e fora das estações e refúgios no continente antártico envolvem um alto consumo de combustíveis e produção de resíduos em solo austral, que mesmo quando devidamente tratados, deixam marcas na biota antártica que ainda não foram claramente estudadas (ATCPs 1993).

Os estudos sobre as fontes de contaminação e os impactos destes no meio ambiente antártico ainda são limitados. Nos ecossistemas terrestres estes estudos são ainda mais escassos, tendo como maior desafio o estabelecimento das relações entre a presença de contaminantes e os efeitos deletérios sobre a biota antártica (Santos et al. 2004)

Torna-se evidente com este ensaio, a fragilidade das formações de musgos nas áreas de degelo da Antártica Marítima. Com a criação de um banco de dados descritivo será possível o monitoramento contínuo das comunidades vegetais da Baía do Almirantado, contribuindo para a conservação vegetação na região. Espera-se assim, que o Índice de Valor Ecológico, como ferramenta de auxilio em estudos de fitossociologia e conservação, possa fornecer subsídios para o planejamento e a organização das atividades científicas dos pesquisadores e militares envolvidos com o Programa Antártico Brasileiro.

\section{REFERÊNCIAS}

ALLISON, J.S. \& LEWIS-SMITH, R.I. 1973. The vegetation of Elephant Island, South Shetland Islands. British Antarctic Survey Bulletin, 33-34: 185-212.

ATCPs. 1993. Protocol on Environmental Protection to the Antarctic Treaty, with Annexes. Polar Record 29(170): 256-275

BEDNAREK-OCHYRA, H.; VÃNA, J.; LEWIS-SMITH, R.I. \& OCHYRA R. 2000. The liverwort of Antarctica. Polish Academy of Science, Institute of Botany, Cracow, 237p.
BRAUN-BLANQUET, J. 1932. Plant Sociology: The study of plant communities. McGraw-Hill, New York, 439p.

HALLINGBÄCK, T. \& HODGETTS, N. 2000. Mosses, liverworts \& hornworts: a status survey and conservation action plan for bryophytes. IUCN, Gland. 106p.

HALLINGBÄCK, T.; HODGETTS, N. \& URMI, E. 1996. How to use the new IUCN Red List categories on bryophytes. Guidelines proposed by the IUCN SSC Bryophyte Specialist Group. Anales del Instituto de Biologia de la Universidad Nacional Autónoma de México, Serie Botánica, 67(1): 47-157.

HU, S-S. 1998. Moss communities types and species diversity of Southern Fields Peninsula (King George Island, South Shetland Islands) Antarctica. Journal Hattori Botanical Laboratory, 84: 187-198.

KANDA, H. \& OHTANI, S. 1991. Morphology of the aquatic mosses collected in lake Yukidori, Langhovde, Antarctica. Proccedings of NIPR Symposium on Polar Biology, 4: 114-122

KANDA, H. 1986. Moss communities in some ice-free areas along the Söya Coast, East Antarctica. Memoirs of Natural. Institute of Polar Research, Special Issue., 44: 229-240.

LARA, F. \& MAZIMPAKA, V. 1998. Sucession of epiphytic bryophytes in a Quercus pyrenaica forest from Spanish Central Range (Iberian Peninsula). Nova Hedwigia, 67: 125-138.

LEWIS-SMITH, R.I. 1972. Vegetation of the South Orkney Island with particular references to Signy Island. British Antarctic Survey Bulletin, 33-34: 89-122.

LEWIS-SMITH, R.I. 1990. Signy Island as a paradigm of biological and environmental change in Antarctic terrestrial ecosystems. Pp. 32-50 In: Kerry, K. R. \& Hempel, G. (eds). Antarctic Ecosystem. Ecological Change and Conservation. Springer-Verlag, Berlin.

LEWIS-SMITH, R.I. 2001. Plant Colonisation Response to climate change in the Antarctic. Folia Fac. Sci. nat. Univ. Masarykianae Brunensis, Geográfica, 25: 19-33.

LONGTON, R.E. 1988. The biology of polar bryophytes and lichens. Cambridge-Sydney, Cambridge University Press, $391 \mathrm{p}$.

MARQUES, J.; HESPANHOL, H.; VIEIRA, C. \& SÊNECA, A. 2005. Comparative study of the bryophyte epiphytic vegetation in Quercus pyrenaica and Quercus robur Woodlands from northern Portugal. Boletin Sociedad Espanhola Briología, 26-27: $75-84$.

MICHEL, R.F.M.; SCHAEFER, C.E.G.R.; DIAS, L.E.; SIMAS, F.N.B.; BENITES,V.M \& SÁ MENDONCA, E. 2006. Ornithogenic Gelisols (Cryosols) from Maritime Antarctica: Pedogenesis, Vegetation and Carbon studies. Soils Science Society of America Journal, 70: 1370-1376. 
OCHYRA, R. 1998. The moss flora of King George Island Antarctica. Polish Academy of Sciences. Cracow, 279p.

OLECH, M. 1996. Human impact on terrestrial ecosystems in West Antarctica. Proccedings of NIPR Symposium on Polar Biology, 9: 299-306.

PUTZKE, J. \& PEREIRA, A.B. 1990. Mosses of King George Island. Pesquisa Antártica Brasileira, 2(1): 17-71.

PUTZKE, J. \& PEREIRA, A B. 2001. The Antarctic Mosses, with special references to the Shetland Islands. Canoas. Ed. ULBRA, 196p.

SANTOS, I.R.; SCHAEFER, C.E.G.R.; SILVA-FILHO, E.V.; ALBUQUERQUE, M.A.; ALBUQUERQUE FILHO, M.R. 2004. Contaminantes antrópicos em ecossistemas antárticos: Estado-da-arte.Pp.95-106.In: SCHAEFER, C.E.G.R.; SIMAS, F.N.B.; ALBUQUERQUE FILHO, M.R. (eds). Ecossistemas costeiros e monitoramento ambiental da Antártica Marítima. Baía do Almirantado, Ilha Rei George. Viçosa, NEPUT.

SCHAEFER, C.E.G.R.; DIAS, L.E.; ALBUQUERQUE, M.A.; FRANCELINO, M.R.; COSTA, L.M. \& RIBEIRO, J.R.E.S. 2004. Monitoramento ambiental e avaliação dos impactos nos ecossistemas terrestres da Antártica Marítima: Princípios e aplicação. Pp. 107-117. In: SCHAEFER, C.E.G.R.; SIMAS, F.N.B.; FILHO, M.R.A. (eds). Ecossistemas costeiros e monitoramento ambiental da Antártica Marítima. Baía do Almirantado, Ilha Rei George. Viçosa, NEPUT.

SIMÕES, J.C.; ARAGONY-NETO, J. \& BREMER, U.F. 2004. Uso de mapas antárticos em publicações. Pesquisa Antártica. Brasileira. 4: 191-197.

SIMÕES, J.C.; FERRON, F.A.; BRAUN, M.; ARIGONYNETO, J. \& AQUINO, F.E. 2001. A GIS for the Antarctic Specially Managed Area (ASMA) of Admiralty Bay, King George Island, Antarctica. Geo-Spatial Information Science (Quarterly), 2: 8-14.

VICTORIA, F.C.; ALBUQUERQUE, M.P. \& PEREIRA, A.B. 2006. Lichen-moss association in plant comunnities of the Southwest Admiralty Bay, King George Island, Antarctica. Neotropical Biology Conservation, 1(2): 84-89.

Submetido em 10/08/2007. Aceito em 14/09/2007. 